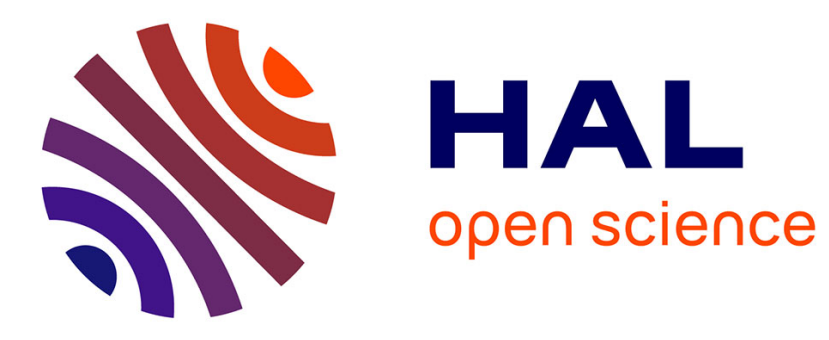

\title{
Crack Detection Based on a Marked Point Process Model
}

\author{
Jennifer Vandoni, Sylvie Le Hégarat-Mascle, Emanuel Aldea
}

\section{To cite this version:}

Jennifer Vandoni, Sylvie Le Hégarat-Mascle, Emanuel Aldea. Crack Detection Based on a Marked Point Process Model. 2016 23rd International Conference on Pattern Recognition (ICPR), Dec 2016, Cancun, Mexico. hal-01664380

\section{HAL Id: hal-01664380 https://hal.science/hal-01664380}

Submitted on 14 Dec 2017

HAL is a multi-disciplinary open access archive for the deposit and dissemination of scientific research documents, whether they are published or not. The documents may come from teaching and research institutions in France or abroad, or from public or private research centers.
L'archive ouverte pluridisciplinaire $\mathbf{H A L}$, est destinée au dépôt et à la diffusion de documents scientifiques de niveau recherche, publiés ou non, émanant des établissements d'enseignement et de recherche français ou étrangers, des laboratoires publics ou privés. 


\title{
Crack Detection Based on a Marked Point Process Model
}

\author{
Jennifer Vandoni, Sylvie Le Hégarat-Mascle and Emanuel Aldea \\ SATIE - CNRS UMR 8029 \\ Paris-Sud University, Paris-Saclay University, France \\ \{jennifer.vandoni, sylvie.le-hegarat, emanuel.aldea\}@u-psud.fr
}

\begin{abstract}
This paper studies the problem of crack detection in images characterized by high gradient backgrounds. We propose an extension of a Marked Point Process model which has been successfully used for wrinkle detection. We show that our method exhibits state of the art results on a difficult image dataset, by proposing a robust trade-off between local analysis approaches, which exploit a limited amount of information around the area of interest, and global reconnection strategies, which aim to detect the crack at image level. Additional tests on a standard dataset show that the proposed method exhibits excellent performance on images with a more uniform background as well, underlining its usefulness in varying contexts.
\end{abstract}

\section{INTRODUCTION}

A crack has the appearance of a discontinuity in a background corresponding to the material (asphalt, concrete, etc.). The detection is hindered by the fact that the background may be not homogeneous, e.g. due to the presence of some lumps or roughness of the material. Besides, since the crack is a thin structure, a major difficulty comes from the fact that it may easily be concealed by noise. Thus we aim to define a process able to distinguish between the gradient response due to background ruggedness and that due to crack presence. Such a distinction may be provided by specific spatial interactions between line segments presenting high gradient response.

Marked Point Processes (MPP) have been proposed for various applications such as road extraction, tree detection or crowd analysis [1]-[4]. For instance, the 'candy' model was proposed [1] to represent a line segment network such that (i) alignments and connections between segments are favored, (ii) unconnected or overlapping line segments are penalized, (iii) crossings are authorized. Recently, [5], [6] proposed a MPP model for wrinkle detection, based on the candy model but different in several senses. In these works, the wrinkles are modeled as sequences of interconnected line segments, with the proposed model relying on adapted prior probability and data likelihood terms. On account of the similarity between crack and wrinkle physical features, in particular the prior model about the type of interactions, we have developed a MPP model for crack detection that nevertheless presents some specificity for our problem.

Many alternative approaches have been explored in the literature, which are either local or global in terms of pixel neighborhood vs. entire image level analysis. An example of the first type of strategy is percolation [7] which classifies a pixel depending on the outcome of a simulated percolation process in its neighborhood. An example of the second kind of approach is [8]. In this latter, maximum paths (in terms of significance) are researched through the image, so that the algorithm of [8] is able to overcome some low contrast parts of the crack in order to detect a global crack. In this work, we argue that the proposed approach based on MPP models offers a robust compromise between a local decision and a global solution which may occasionally drift from a regular crack model. A second contribution of our work is that, with respect to wrinkle detection, we extend the MPP model in order to cope with structures which exhibit less alignment and more complex interactions than wrinkles.

\section{PROPOSED APPROACH BASED ON MPP MODEL}

A Marked Point Process handles a random set of elements, such that each element has the form of a couple consisting of a point and a vector of parameters, called a 'mark'. Usually, the point represents the position of an object of interest whereas the mark supplements its geometric description. All the points $\mathbf{p}_{\mathbf{i}}, i \in\{1, \ldots, n\}$ are included in a compact area that is the image domain, $\mathcal{F} \in \mathbb{R}^{2}$. Then, the marks associated to the elements of $\mathcal{F}$ provide the geometrical features that allow us to specify the object and to evaluate its likelihood from its appearance in the data image. It also allows for the analysis of the interactions between marked points in order to evaluate a global solution (configuration of marked points on $\mathcal{F}$ ) versus a prior about favorable configurations.

For instance, a crack will be represented by a group of line segments that are the marked points, themselves represented by a point (the segment's center in our case, but it could equivalently have been one extremity) and a mark including two features, namely the orientation and the length of the line segment. Then, each marked point defines a set of image pixels so that the likelihood for these pixels to belong to a crack can be computed (like in any probabilistic classification), corresponding to the 'data fidelity term'.

Since a crack is more or less a fine rectilinear structure, the favorable configurations correspond to close and aligned marked points. Then, the marked point interactions derive from the point orientation (given in the mark) and its location (given by the point coordinates and the length given in the mark). These geometric interactions provide the prior probability of the configuration (spatial arrangement), corresponding to the 'prior term'. Considering a given configuration of marked 
points, its probability is finally estimated by the product between the likelihood term and a prior probability.

Specifically, let us denote $w_{i}=\left(\mathbf{p}_{i}, \mathbf{m}_{i}\right)$ a line segment, where $\mathbf{p}_{i}=\left(x_{i}, y_{i}\right)$ encodes the center of the segment, while the mark $\mathbf{m}_{i}=\left(l_{i}, \theta_{i}\right)$ encodes its length and orientation. $w_{i}$ is a realization of the random set of variables taking its values in $\mathcal{S} \times\left[l_{\text {min }}, l_{\text {max }}\right] \times\left[\theta_{\text {min }}, \theta_{\max }\right]$, with $\mathcal{S}$ the image lattice and $\left[l_{\min }, l_{\max }\right]$ and $\left[\theta_{\min }, \theta_{\max }\right]$ the continuous intervals for parameters $l_{i}$ and $\theta_{i}$, respectively. The optimal line segment configuration should then maximize the posterior probability defined by the MPP model specified in the next section.

\section{A. MPP model for crack detection}

Assuming $\mathbf{w}$ is the realization of a Gibbs random set, the posterior probability density is $f(\mathbf{w}) \propto \beta^{n(\mathbf{w})} \exp [-U(\mathbf{w})]$ with $\beta$ the intensity of the point process and $U(\mathbf{w})$ called the energy function that, in case of pair interactions, writes

$$
U(\mathbf{w})=\sum_{i=1}^{n} U_{d}\left(w_{i}\right)+\sum_{1 \leq i<j \leq n} U_{p}\left(w_{i}, w_{j}\right) .
$$

In [5], the data fidelity term is a sum over the line segment pixels of the response of the Laplacian of Gaussian (LoG) filter. In [6], the authors propose rather to consider the directional response using a steerable filter [9] defined so that a filter of arbitrary orientation is obtained as a linear combination of a set of basis filters. If, as in [6], we consider the second derivative basis at pixel $s, \mathbf{G}_{2}(s)=\left[\frac{\partial^{2} g(s)}{\partial x^{2}} \frac{\partial^{2} g(s)}{\partial x \partial y} \frac{\partial^{2} g(s)}{\partial y^{2}}\right]^{\top}$ where $g(s)$ is the Gaussian 2D function and $\mathbf{v}^{\top}$ denotes the transpose vector of vector $\mathbf{v}$, then the interpolating function of orientation is [9]:

$$
\mathbf{G}_{2, \theta}(s)=\left(\begin{array}{lll}
\cos ^{2} \theta & -\sin 2 \theta & \sin ^{2} \theta
\end{array}\right) \mathbf{G}_{2}(s) .
$$

In our case, we follow the idea of [6] that the data fidelity term takes into account the line segment orientation. However, instead of computing the response corresponding to the 'exact' line segment orientation, we approximate this latter at $\pm \frac{\pi}{16}$. This allows us to precompute the images $I_{2, \tilde{\theta}}=\mathbf{G}_{2, \tilde{\theta}} * I$ of the filter response for $\tilde{\theta} \in\left\{0, \frac{\pi}{8}, \frac{\pi}{4}, \frac{3 \pi}{8}, \frac{\pi}{2}, \frac{5 \pi}{8}, \frac{3 \pi}{4}, \frac{7 \pi}{8}\right\}$ and to obtain the data fidelity term value directly from these images. Specifically, for a line segment $w_{i}$ with $\theta \cong \tilde{\theta}$, the data term $U_{d}\left(w_{i}\right)$ is the sum of $w_{i}$ pixel values in image $I_{2, \tilde{\theta}}$.

For the prior term, we modify the energy proposed in [5], [6] to take into account some specificities of crack detection: if the basic idea remains to favor the aligned close line segments, we stand out from previous work by the neighborhood definition and the weight of each neighbor in the prior energy definition. The aim of this prior energy term is to encourage smoothly connected lines while rejecting occurrences of abrupt intersection or overlapping between line segments. We first define the set of interacting segments (called 'neighbors') with given segment $w_{i}$ : a segment $w_{j}$ is a neighbor of $w_{i}$ if the distance $\operatorname{dist}_{t}\left(w_{i}, w_{j}\right)$ between $w_{i}$ and $w_{j}$ is lower than the sum of their half lengths, which may be interpreted as an adhoc adaptive threshold:

$$
w_{i} \sim w_{j} \text { if } \operatorname{dist}_{t}\left(w_{i}, w_{j}\right) \leq \frac{l_{i}+l_{j}}{2} .
$$

In [5], [6], segment distance was simply the distance between their centers. However, such a distance fails in taking into account the respective orientations of the segments and depends on the 'fragmentation' of the segments (i.e. the number of subsegments in which a long segment is subdivided). Thus, in our case, dist $_{t}\left(w_{i}, w_{j}\right)$ is defined from the actual distance between segments $\operatorname{dist}\left(w_{i}, w_{j}\right)$, that is the minimum distance between any couple of points belonging to $w_{i}$ and $w_{j}$, respectively. $\operatorname{dist}\left(w_{i}, w_{j}\right)$ is computed according to [10]. Then, in order to cope with segment fragmentation, we apply a transitivity property on previous distances: having computed dist $\left(w_{k}, w_{l}\right), \forall\left(w_{k}, w_{l}\right) \in \mathbf{w} \times \mathbf{w}$, $\operatorname{dist}_{t}\left(w_{i}, w_{j}\right)=\min _{w_{k} \in \mathbf{w}} \operatorname{dist}\left(w_{i}, w_{k}\right)+\operatorname{dist}\left(w_{k}, w_{j}\right)$. The distances $\operatorname{dist}_{t}\left(w_{i}, w_{j}\right)$ are initialized with $\operatorname{dist}\left(w_{i}, w_{j}\right)$ and then updated iteratively, until convergence.

Two neighbors $w_{i}$ and $w_{j}$ can interact either 'repulsively' or 'attractively'. In [5], the prior energy term only considers repulsive neighbors (defined as belonging to a spatial rejection area) in order to avoid some congested areas having overlapping line segments, and does not penalize isolated line segments. In [6], attractive interaction occurs only between sufficiently aligned and smoothly connected segments:

$w_{i} \sim{ }^{a} w_{j}$ if $\left\{\begin{array}{l}\theta_{i j}=\min \left(\left|\theta_{i}-\theta_{j}\right|, \pi-\left|\theta_{i}-\theta_{j}\right|\right) \leq \theta_{\max } \\ d_{i j}=\operatorname{dist}_{t}\left(w_{i}, w_{j}\right) \leq d_{\max }\end{array}\right.$

For each couple of attractive neighbors, the prior energy term of [6] maps the marks $\left(\mathbf{m}_{i}, \mathbf{m}_{j}\right)$ onto the interval $[0,1]$, thus penalizing neighbors which are farther and less aligned. However, the term proposed in [6] penalizes multiple neighbors. Since in our case, we want to favor multiple neighbors, e.g. one neighbor at each extremity, we modify this term in two ways: firstly, each couple of neighbor marks $\left(\mathbf{m}_{i}, \mathbf{m}_{j}\right)$ are mapped onto the interval $[-1,0]$ and secondly, previous values are weighted by the length of the line segments:

$$
\begin{aligned}
\forall\left(w_{i}, w_{j}\right) & \mid w_{i} \sim^{a} w_{j}, \\
K\left(l_{i}, l_{j}\right)= & \frac{1}{2}\left[\frac{1}{1+e^{\lambda-l_{i}}}+\frac{1}{1+e^{\lambda-l_{j}}}\right], \\
U_{a}\left(w_{i}, w_{j}\right)= & -K\left(l_{i}, l_{j}\right)\left[\phi\left(d_{i j}, d_{\max }\right)+\right. \\
& \left.\phi\left(\theta_{i j}, \theta_{\max }\right)\right],
\end{aligned}
$$

with $\lambda=d_{\max }$ in our case and $\phi\left(x, x_{\max }\right)=$ $\left(1-\left(\frac{x}{x_{\max }}\right)^{2}\right) \times\left(1+x^{2}\right)^{-1}$ the Quality Function used in [6] to map $d_{i j}$ or $\theta_{i j}$ values onto $[0,1]$. Finally, the prior energy term is:

$$
\begin{aligned}
U_{p}(\mathbf{w})= & \beta_{0} \sum_{\substack{i \mid \forall j \neq i, w_{i} \nsim w_{j}}} K\left(l_{i}, l_{j}\right)+\beta_{r} \sum_{\substack{(i, j) \mid j \neq i, w_{i} \sim w_{j}, w_{i} \nsim^{a} w_{j}}} K\left(l_{i}, l_{j}\right)+ \\
& \beta_{a} \sum_{\substack{(i, j) \mid \\
w_{i} \sim^{a} w_{j}}} U_{a}\left(w_{i}, w_{j}\right),
\end{aligned}
$$

with $\nsim$ denoting the absence of neighborhood relationship, $\beta_{0}$, $\beta_{r}$ and $\beta_{a}$ three parameters of the MPP model. The choice of these parameter values depends on the weight given to prior 


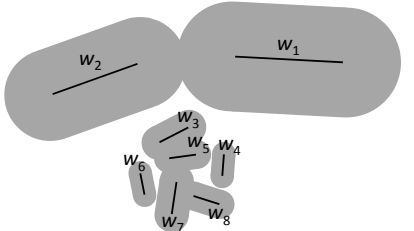

(a)

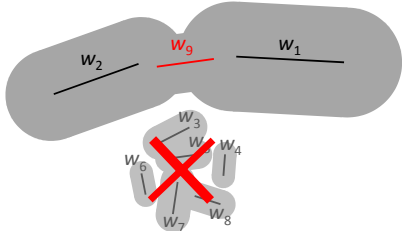

(b)
Fig. 1. Toy example with segment neighborhoods shown in gray. For $\theta_{\max }=$ $\frac{\pi}{4}$, on the left, 3 out of the 4 interactions are repulsive and 2 line segments are isolated, whereas, on the right, the 2 segment interactions are attractive.

energy relatively to data fidelity and on the strength with which we want to reject the isolated segments or the ones exhibiting repulsive interaction.

Figure 1 shows a configuration example with nine line segments and four neighborhood relationships (the neighborhood areas are represented in gray): $w_{1} \sim w_{2}, w_{3} \sim w_{5}, w_{5} \sim w_{7}$ and $w_{7} \sim w_{8}$ (in this simple case there is no supplementary neighbourhood relationship added by transitivity), so that $U_{p}(\mathbf{w})=2 \beta_{0}+3 \beta_{r}+\beta_{a}\left(U_{a}\left(w_{1}, w_{2}\right)\right)$ for $\theta_{\max }=\frac{\pi}{4}$.

\section{B. MPP algorithm for crack detection}

The Reversible Jump Markov Chain Monte Carlo (RJMCMC) was proposed [11] to allow comparison (and 'jump') between different configurations possibly having different dimensions, i.e. numbers of line segments for our case.

Let us first recall that modifying one line segment $w_{i}$ has only an impact on a finite subset of energy terms: the data fidelity term of the modified segment itself $U\left(w_{i}\right)$ and the prior terms of line segments belonging to the disjunction of the sets of neighbors of $w_{i}$ before and after its modification (one of these sets being possibly empty when the $w_{i}$ modification is a 'death' or a 'birth'). Then, the computation of the difference between global energy after and before modifying $w_{i}$ boils down to the sum of the differences on impacted energy terms.

Like [5], we use a set of transition kernels that help the convergence of the model toward the solution. However, in [5] the initialization was good enough (due to the fact that the problem is different, the authors start from a specific initialization) to require only transitions which deal with the addition of new segments relying on already existing segments or extending (ending) them. In our case, the considered transitions are:

- Birth of a new line segment connecting two other line segments that are repulsive because of their distance;

- Death of a line segment that is isolated (distance with the closest line segment is greater than the sum of the segment lengths);

- Birth of a new line segment connecting two other line segments $w_{i}$ and $w_{j}$ that are aligned $\left(\theta_{i j}<\theta_{\max }\right)$;

- Birth of a new line segment connecting at least one extremity of another line segment that belongs to the set of the large connected components (clusters of connected line segments);
- Death of a line segment belonging to the repulsive area of a another line segment (with repulsive area of $w_{i}$ being equal to the dilation of $w_{i}$ by a linear structuring element perpendicular to $w_{i}$ and of half length);

- For a given segment, modification of one of its extremities by moving it in its 8-connectivity neighborhood.

\section{Preprocessing}

The preprocessing aims at obtaining an image on which the previous MPP model corresponds better to the crack. Two phenomena penalize the crack detection. The first one is the presence of shadows whose borders may present a high response to derivative filters and whose low radiometric values may be close to some crack pixel values. The second one is the presence of some asperities or textons due to the important roughness of the material. This is in particular the case of road surfaces or rough plaster walls. These asperities look like a texture that provides both unwanted response to derivative filter and some dark pixels because of micro-shadows.

The shadow removal is simply done using background subtraction technique. To estimate the background first a morphological closing is applied to the original image to remove the thin dark structures (cracks and asperities) and then a Gaussian filtering $(\sigma=5)$ is applied to recover large scale variations on the image. Then, denoting $I$ the original image, $f_{G(\sigma)}$ the Gaussian filter, * the convolution, and $\phi()$ the morphological closing operator: $I_{-B}=I-f_{G(\sigma)} * \phi(I)$.

The textons removal is more difficult. Due to light/shadow phenomenon, they present a dark part and a bright part. To remove at least the bright part, we perform a functional geodesic reconstruction of $I_{-B}$ from its copy lowered of the Otsu threshold value. Let $\tilde{I}$ denote the obtained image.

Finally, as in [8], an image composition is computed that is a linear combination between $\tilde{I}$ and the maximum ratio value of its derivative response in one direction and the perpendicular one (see [8] for further details).

\section{Postprocessing}

A postprocessing step is beneficial for precise crack detection for two reasons. Firstly, when segment ends are detected reliably, a min-cost path algorithm between them follows accurately the actual crack. Once this segment relaxation step is performed, we compute the average $\mu$ of the crack pixels. Thus, for long (more than 15px) crack segments with more than two ends, we relax first the path between the most distant ends, then we check all the secondary smaller paths against $\mu$ and we prune them if they are not dark enough. Then we identify and try to reconnect independent crack ends by new min-cost paths; we accept conservatively only the paths which have an average value lower than $\mu$. This step is important, as it allows us to apply high-quality connections in some areas which were not favored by MPP, such as short sinuous paths between two almost perpendicular crack segments. Finally, in a last step, we try to connect the path ends with the border of the image in order to recover potential peripheral crack parts, and we apply the same test based on $\mu$. 

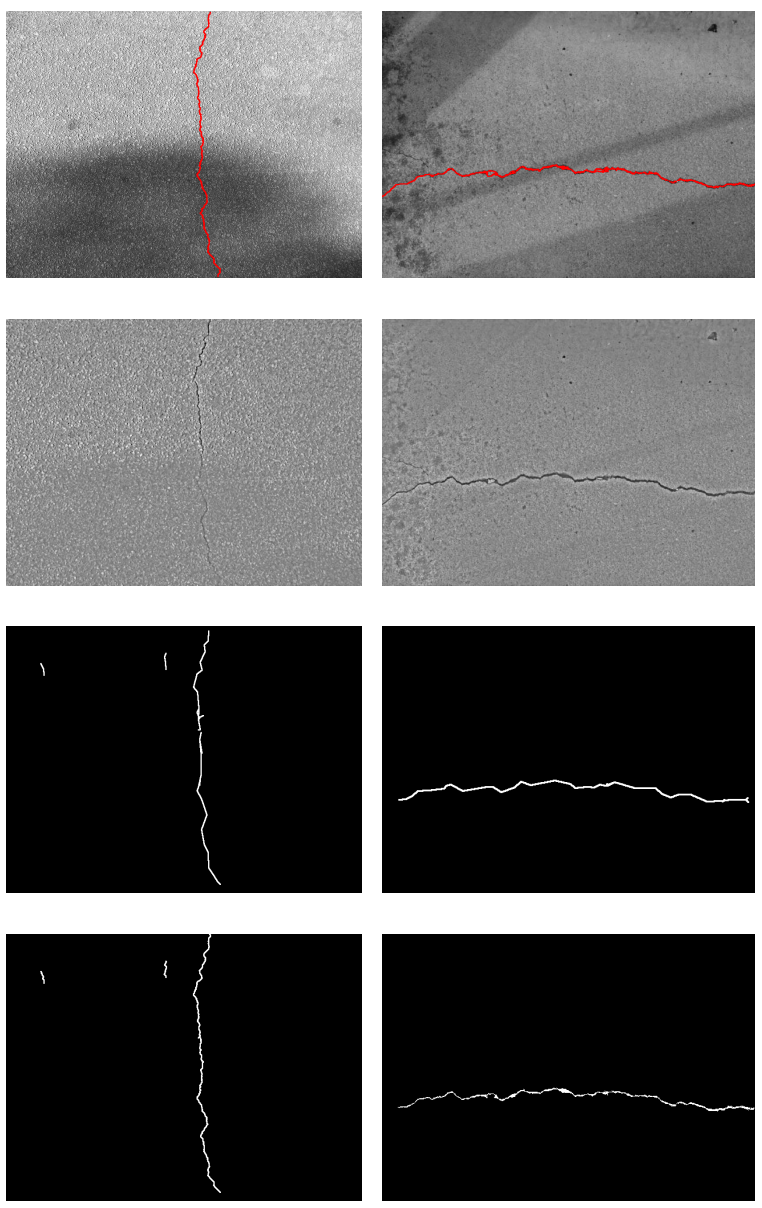

Fig. 2. Crack detection steps on both datasets. Row 1: original images with ground-truth cracks highlighted in red. Row 2: shadow removal results. Row 3: cracks detected as segments with proposed MPP. Row 4: final results after post-processing.

Note that both preprocessing and postprocessing are required for the efficiency of the proposed approach, as they cope with physical features independent of the MPP part (radiometric features and connectivity, respectively).

\section{RESULTS}

The crack dataset proposed by the authors of [12] has been employed for the experimental tests, from now on referred to as CTD. It fits particularly well the application since asphalt textons and other degradation artifacts are commonly present. In order to show the robustness of the method against different types of surfaces, i.e. noisy concrete surface, also the dataset proposed in [13] has been considered, referred to as $\mathrm{CnD}$.

Figure 2 shows two examples of crack detection using the proposed approach on both datasets. Removing the shadows allows for a better detection of the crack even if the difference of contrast with respect to the background is not so high. Then, the MPP algorithm is applied and the segments which compose the cracks are well detected as they comply with the energy minimization statement. In order to better follow the behavior of the actual cracks and to connect close segments, the post- processing using path minimization resulted to be particularly well suited since it exploits intensity local minima to refine the actual path of the crack, which is usually rather jagged. Please note that only for $\mathrm{CnD}$, after the described post-processing, a thickening has been applied, as the ground truth cracks come with varying widths.

In order to validate our new approach, we compared it with the percolation based local method of [7], and with NFA [8]. Figure 3 shows the comparison on four meaningful images of CTD. Please note that for fairness in the second row the rough results of MPP segments are shown in red, while the final results are superimposed in yellow so that it is easy to see how the post-processing step is able to relax the MPP model following better the actual cracks. Besides, in green are shown the new connection paths added by the post-processing. The percolation method, while having the advantage of being fast, is not able to discriminate well enough between actual cracks and asphalt textons on this particular type of images, since it has been designed mostly for concrete surfaces, and overall it has difficulties on noisy surfaces. The NFA approach exploits the NFA criterion to select the paths that are the most likely to represent the cracks. It is reasonably appropriate for this type of surface, as in most cases it is able to precisely find crack paths between intensity local minima due to the asphalt texture. However, in some cases this becomes a drawback, as it may find very elongated structures between textons which are erroneously considered as cracks. NFA has the tendency to overestimate the cracks paths; conversely, by using MPP we are able to reduce the number of false positive segments, or at least their lengths, at the same time almost without influencing true detections. Another advantage of the new MPP approach is that it overcomes the NFA results in presence of alligator cracks (like in 4th and 5th columns), which are usually the most difficult ones. Indeed, the particular statement of energy minimization that encourages segments with multiple neighbors is the key, with respect to NFA which focuses more on obtaining long paths.

As already said, this work focuses more on crack detection in presence of particular structures like asphalt textons in the images. However, it is worth performing a comparison also on $\mathrm{CnD}$, to show the robustness of the method against different surfaces. In particular, Fig. 4 proves what we expected: percolation works reasonably well on this type of images, being able to detect also thinner cracks, even if sometimes false positives are present. NFA and MPP are still able to detect the most important wider cracks, and they also present less false detections. However, they generally miss the thinner cracks, and this correlates with our aim of being more robust to background imperfections.

In order to compare quantitatively the results of these different approaches on both datasets, precision and recall parameters are computed at different dilation scales, as in [8]: true positives and false positives are computed comparing the detection results with incrementally dilated ground-truth, while false negatives are computed comparing the ground-truth with an incrementally dilated version of the detection results. 

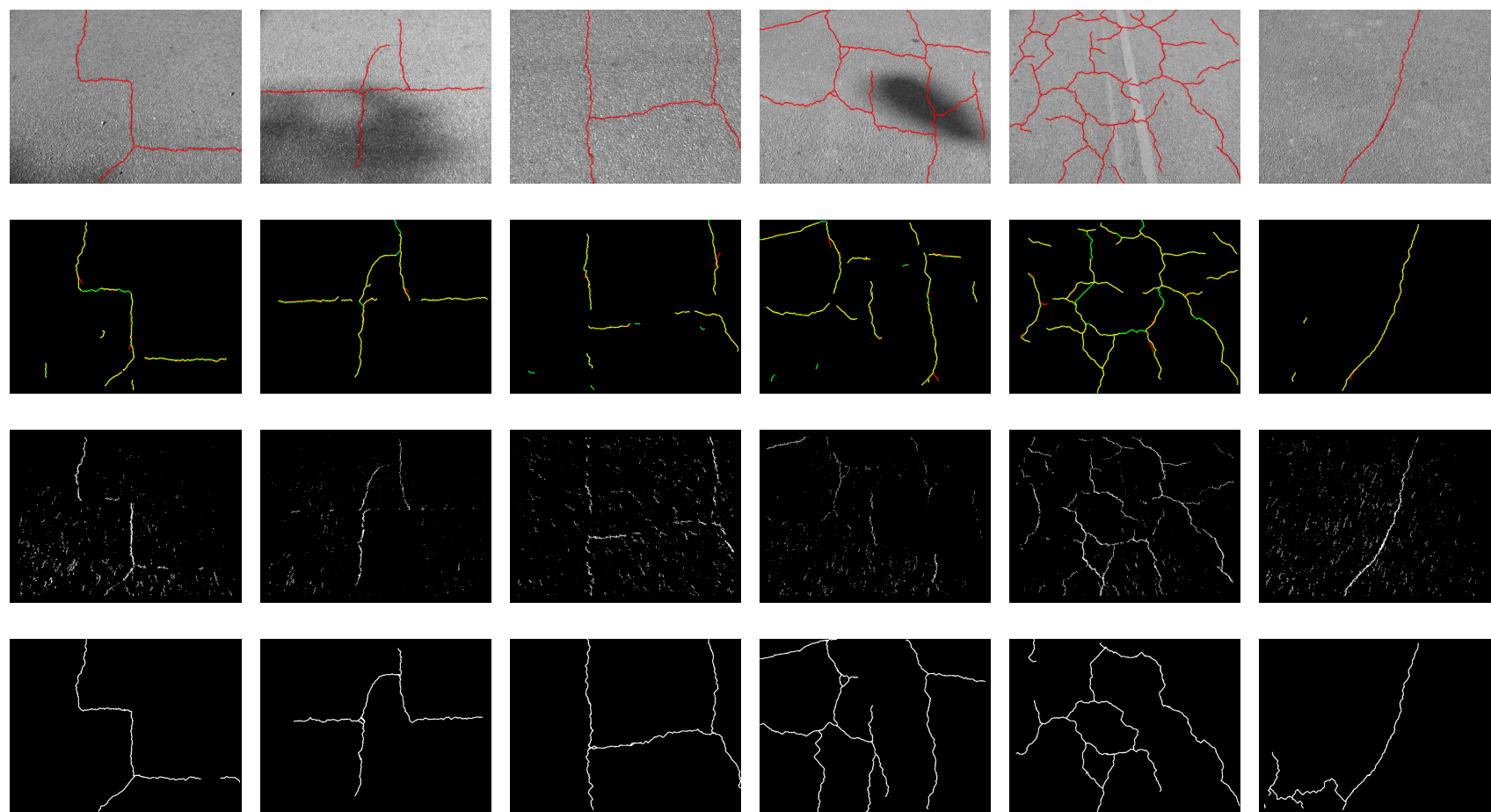

Fig. 3. Comparison between different crack detection methods on CTD. Row 1: original images with ground-truth cracks highlighted in red. Row 2: results with proposed MPP. Row 3: results with percolation. Row 4: results with NFA.
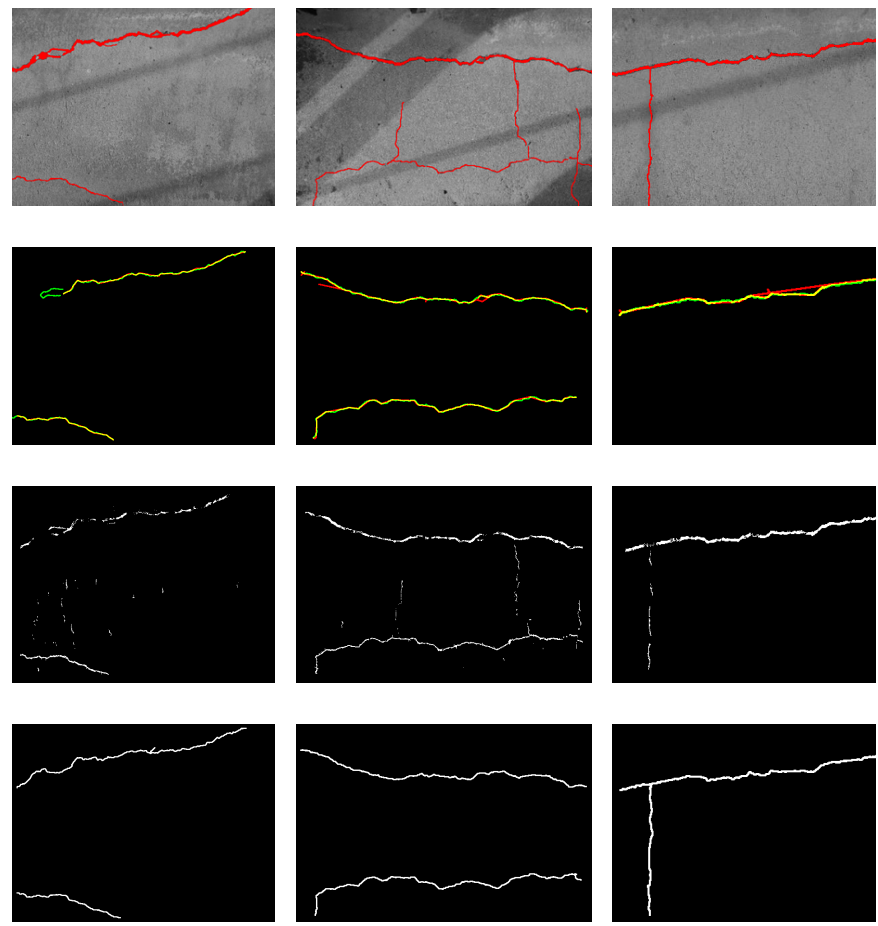

Fig. 4. Comparison between different crack detection methods on $\mathrm{CnD}$. Row 1: original images with ground-truth cracks highlighted in red. Row 2: results with proposed MPP. Row 3: results with percolation. Row 4: results with NFA.

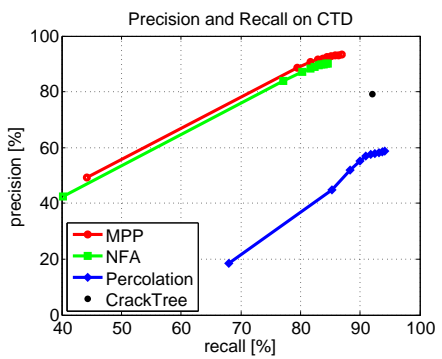

(a)

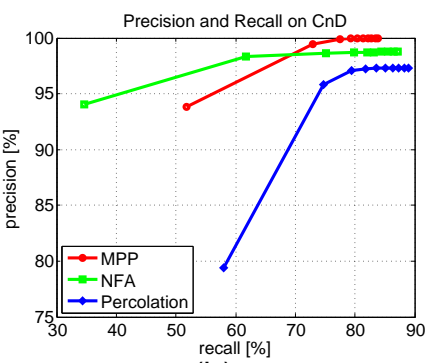

(b)
Fig. 5. Precision-recall plots on a) CTD and b) CnD.

Specifically, at scale 0 no dilation is performed neither in ground-truth nor in the results, while dilation scale 9 means that we allow up to 9px distance between ground-truth and detected cracks. This allows us to distinguish between errors due to a slight mislocation of the crack and actual nondetections or false detections. Figure 5a shows the precisionrecall plot at different scales on the CTD, while Fig. 5b shows the plot on $\mathrm{CnD}$. We may notice how MPP overtakes percolation precision on both datasets, and this agrees with the fact that percolation is less robust against false positives. MPP algorithm is able to exceed NFA precision and recall on CTD. Indeed, as we said, MPP usually does not find false long segments like NFA, allowing for a better precision, and at the same time it works particularly well in presence of segments with multiple neighbors, allowing for a better reconstruction of the crack and therefore higher recall. In particular, considering a reasonable dilation scale of $2 \mathrm{px}$ on $\mathrm{CTD}$, precision and recall 


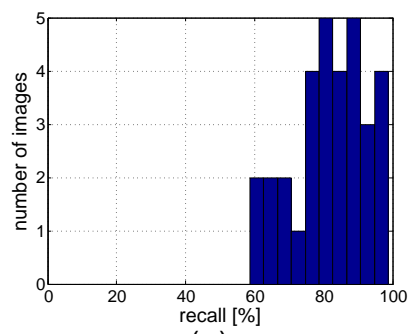

(a)

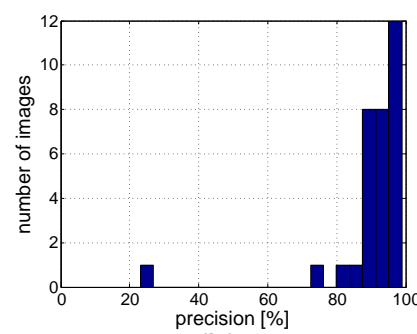

(b)
Fig. 6. a) Recall and b) precision histograms on a subset of 32 images of CTD.
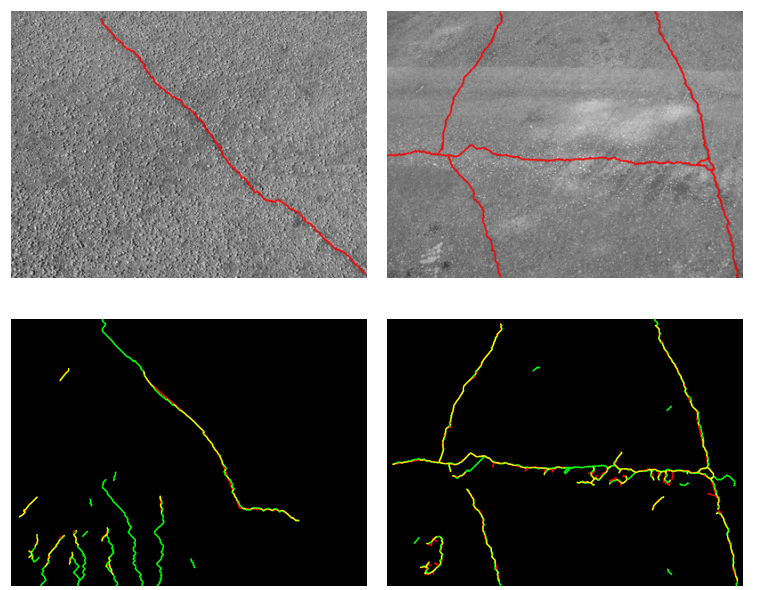

Fig. 7. Crack detection on difficult images due to the presence of aligned noise. Row 1: original images with ground-truth cracks highlighted in red. Row 2: results with MPP.

are quite high, reaching on average respectively values of $90.5 \%$ and $81.6 \%$. Nevertheless, allowing just $1 \mathrm{px}$ mislocation of the cracks, MPP exceeds NFA also on CnD, even if looking at the overall performance it is possible to notice that NFA has better recall since in some cases it succeeds in detecting thin cracks, as in the last image of Fig. 4.

We also compare our MPP approach and the CrackTree algorithm proposed in [12], as both are based on the same idea of determining seeds and then trying to connect them in longer structures using segments. We found out that our results are comparable with CrackTree. In fact, considering CTD and allowing for 2px mislocation, they obtain an F-measure of 0.85 compared to 0.86 of the proposed method, 0.83 of NFA and 0.65 of percolation.

Lastly, in order to identify the main difficulties of the MPP approach, we created two histograms for precision and recall values at dilation scale $2 \mathrm{px}$ on a subset of 32 images of CTD, shown in Fig. 6. The recall histogram is in agreement with our expectations: with a min value of $58.6 \%$ and a max value of $98.7 \%$, the average value is around $80 \%$. Conversely, looking at the precision plot in Fig. $6 b$, we note that the average value is strongly penalized by only 2 (out of 32 ) values.

Figure 7 investigates more thoroughly two images which are challenging for our method. The reason of this performance drop is easily explainable since these two images present aligned noise, which can be misleading and bring false detection, resulting in lower precision. However, we can also notice a trade-off due to the post-processing step in the first image. While improving the detection and the accuracy of the crack in the upper part, post-processing tends to connect false positive segments in the lower part of the image, thus having a notable impact on the final result.

\section{CONCLUSION}

We have presented a MPP model based method for crack detection which benefits at the same time from a local analysis related to the data fidelity term, and from a more extended spatial inference provided by the prior energy term. Thanks to the coupling between the two terms, the proposed method is able to cope with detections on noisy surfaces while at the same time avoiding false detections specific to min-cost path based methods. A final post-processing step allows us to relax the MPP model and to approach more accurately the crack configuration. Regarding the validation of the method, we show that we obtain state of the art results on two different datasets of varying difficulty. The perspectives of our work relate to the improvement of the computational cost, and to the implementation on hybrid architectures for real-time applications.

\section{REFERENCES}

[1] R. Stoica, X. Descombes, and J. Zerubia, "A gibbs point process for road extraction from remotely sensed images," International Journal of Computer Vision, vol. 57, no. 2, pp. 121-136, 2004.

[2] C. Lacoste, X. Descombes, and J. Zerubia, "Point processes for unsupervised line network extraction in remote sensing," Pattern Analysis and Machine Intelligence, IEEE Transactions on, vol. 27, no. 10, pp. 1568-1579, 2005.

[3] G. Perrin, X. Descombes, and J. Zerubia, "A marked point process model for tree crown extraction in plantations," in Image Processing (ICIP), IEEE Int. Conf. on, vol. 1. IEEE, 2005, pp. I-661.

[4] W. Ge and R. T. Collins, "Marked point processes for crowd counting," in Computer Vision and Pattern Recognition (CVPR), IEEE Int. Conf. on. IEEE, 2009, pp. 2913-2920.

[5] N. Batool and R. Chellappa, "Modeling and detection of wrinkles in aging human faces using marked point processes," in Computer VisionECCV 2012. Workshops and Demonstrations. Springer, 2012, pp. 178 188.

[6] S.-G. Jeong, Y. Tarabalka, and J. Zerubia, "Marked point process model for facial wrinkle detection," in Image Processing (ICIP), 2014 IEEE International Conference on. IEEE, 2014, pp. 1391-1394.

[7] T. Yamaguchi and S. Hashimoto, "Fast crack detection method for largesize concrete surface images using percolation-based image processing," Machine Vision and Applications, vol. 21, no. 5, pp. 797-809, 2010.

[8] E. Aldea and S. Le Hégarat-Mascle, "Robust crack detection for unmanned aerial vehicles inspection in an a-contrario decision framework," Journal of Electronic Imaging, vol. 24, no. 6, pp. 061 119-061 119, 2015.

[9] W. T. Freeman and E. H. Adelson, "The design and use of steerable filters," IEEE Transactions on Pattern Analysis and Machine Intelligence, vol. 13, pp. 891-906, 1991.

[10] V. J. Lumelsky, "On fast computation of distance between line segments," Information Processing Letters, vol. 21, no. 2, pp. 55-61, 1985.

[11] P. J. Green, "Reversible jump markov chain monte carlo computation and bayesian model determination," Biometrika, vol. 82, no. 4, pp. 711732, 1995.

[12] Q. Zou, Y. Cao, Q. Li, Q. Mao, and S. Wang, "Cracktree: Automatic crack detection from pavement images," Pattern Recognition Letters, vol. 33, no. 3, pp. 227-238, 2012.

[13] Y. Fujita and Y. Hamamoto, "A robust automatic crack detection method from noisy concrete surfaces," Machine Vision and Applications, vol. 22, no. 2, pp. 245-254, 2011. 